\title{
Diversidad genérica de Trichoptera (Insecta) en dos microcuencas del Páramo Rabanal (Cundinamarca-Boyacá, Colombia)
}

\author{
Ivonne T. Latorre-Beltrán ${ }^{1}$, Rodolfo Novelo-Gutiérrez ${ }^{2} \&$ Mario E. Favila $^{3}$
}

1. Investigadora independiente. Carrera 28B Nº63F 88, Bogotá, Colombia; ivonnebiology@gmail.com

2. Instituto de Ecología A.C. Red de Biodiversidad y Sistemática. Carretera antigua a Coatepec \# 351, E1 Haya, 91070 Xalapa, Veracruz, México; rodolfo.novelo@inecol.edu.mx

3. Instituto de Ecología A.C. Red de Ecoetología. Carretera antigua a Coatepec 351, El Haya, Xalapa 91070, Veracruz, México; mario.favila@inecol.edu.mx

Recibido 12-XII-2013. Corregido 20-I-2014. Aceptado 13-II-2014.

\begin{abstract}
Generic diversity of Trichoptera (Insecta) of Paramo Rabanal (Cundinamarca-Boyacá, Colombia). Trichopterans are considered an important and diverse biotic element in continental aquatic ecosystems. The purpose of this study was to evaluate the assemblages of the order Trichoptera in two subwatersheds with a gradient of disturbance. Four sampling events were conducted in two subwatersheds in the Eastern Mountain Range of the Colombian Andes. For the analysis we used rarefaction curves, Bray-Curtis' Index and Partitioning Diversity and total richness and Shannon's diversity as metrics. Although total richness was similar between both subwatersheds, abundance was always highest in streams within the conserved subwatershed. Each subwatershed was dominated by different genera, except Ochrotrichia, which was abundant at all sites. Alpha diversity was similar among streams in the conserved watershed, while a reduction in diversity potentially associated with the disturbance gradient was observed in streams of the disturbed subwatershed. Beta diversity $\left({ }^{0} D_{\beta}\right.$ and $\left.{ }^{l} D_{\beta}\right)$ between subwatersheds and among conserved streams was similar, while in disturbed streams a similar gradient to that of alpha diversity was found. The similitude analysis clustered streams according to their conservation status. Differences found in trichopteran assemblages do confirm that the use of their attributes is adequate to assess the conservation status of stream ecosystems. Rev. Biol. Trop. 62 (Suppl. 2): 97-110. Epub 2014 April 01.
\end{abstract}

Key words: diversity, Trichoptera, paramo Rabanal, disturbance, conserved, partitioning diversity.

\footnotetext{
En reconocimiento a la profesora María Eugenia Rincón $H$., que su esfuerzo por entender los ecosistemas acuáticos colombianos, especialmente su Tricopterofauna, trascienda a través de sus enseñanzas.
}

Los páramos son considerados biomas exclusivos de las montañas de la región Neotropical que se distribuyen a manera de islas sobre las cadenas montañosas de Costa Rica, Panamá y el norte de los Andes (Cleef, 1978). Estas regiones están dominadas por vegetación de tipo arbustiva ubicadas en la parte más alta de las montañas, entre el límite superior del bosque alto andino (3200-3 $800 \mathrm{~m}$ de altitud) y el límite inferior de las nieves perpetuas
(4400-4 700m de altitud) (Cuatrecasas, 1989). Los sistemas acuáticos en los páramos colombianos son un interesante caso de estudio, ya que han sido sometidos a procesos intensivos de transformación y degradación, causados principalmente por el cambio de uso del suelo, como consecuencia del desarrollo de actividades como la ganadería, la agricultura y la reforestación con plantas introducidas como eucalipto y pino, principalmente (Rivera, 
2001). La complejidad climatológica y topográfica de este bioma ha favorecido la aparición de comunidades con un alto grado de endemismo, incluso detectable en grupos de insectos como los tricópteros, donde se estima que cada sistema montañoso alberga una o dos especies endémicas (Holzenthal \& Flint, 1995).

Los trabajos sobre diversidad del orden Trichoptera en zonas de páramos de Colombia son escasos o forman parte de trabajos no publicados. Posada \& Abril (2008) realizaron un estudio en el páramo de Frontino (Antioquia, Colombia) en sistemas lóticos y lénticos. En los sistemas lóticos los autores encontraron ocho géneros de tricópteros, aunque las curvas de acumulación sugieren que para estos sistemas el muestreo fue insuficiente. En cuanto a estudios en alta montaña que comparen la diversidad de macroinvertebrados entre sistemas perturbados y no perturbados existe uno realizado en el centro de Brasil en el que se encontró que las actividades antrópicas determinaron los patrones de diversidad (Bispo \& Oliveira, 2007).

La estructura actual del paisaje en el sector sur del complejo de páramos Rabanal corresponde a un mosaico en el que predomina vegetación herbácea, tanto en el páramo natural, como en las zonas afectadas por la actividad humana (Morales et al., 2007). Esta variabilidad permite evaluar la respuesta de la comunidad de macroinvertebrados al estado de conservación del páramo, utilizando para ello arroyos que se encuentran en áreas conservadas o bien afectadas por la actividad humana. De acuerdo con la familia o género al que pertenezcan, los tricópteros poseen distintos rangos de tolerancia a la contaminación del agua y a la alteración del hábitat, siendo uno de los órdenes de macroinvertebrados más sensibles a dichas condiciones y usados comúnmente en estudios de biomonitoreo o de evaluación de calidad de agua (Springer, 2010).

El propósito de este trabajo fue evaluar y comparar la riqueza de géneros, composición y abundancia de las comunidades del orden Trichoptera encontrados en los arroyos de dos microcuencas con diferente grado de perturbación por actividades antrópicas en un sector del páramo Rabanal (CundinamarcaBoyacá, Colombia). Se seleccionó este orden debido a que está presente prácticamente en todos los sistemas dulceacuícolas, su diversidad taxonómica es alta y relativamente bien conocida (Bispo \& Leandro, 2007). Además, ocupa microhábitas muy variados y participa en diferentes procesos ecológicos tales como descomposición de hojarasca por parte de especies filtradoras y fragmentadoras, control de la productividad primaria debido a que se alimentan de algas. Los tricópteros son también parte de la dieta de otros organismos como peces, ranas, aves, murciélagos y arañas (Springer, 2010). Su diversidad y poca tolerancia a sistemas contaminados hacen de este orden un excelente indicador de calidad de agua y de integridad ecológica (Rosenberg \& Resh, 1993). Conociendo esto, sugerimos que la diversidad de la comunidad de tricópteros a distintas escalas espaciales (entre microcuencas y arroyos) refleja el estado de conservación de las microcuencas que habitan. Si es así, se espera que tanto el número de géneros como su abundancia sea menor en los arroyos de la microcuenca ubicados en el área perturbada. En cuanto a la partición de los componentes de la diversidad se espera que la magnitud de recambio de géneros sea menor en los arroyos de la microcuenca perturbada que en la conservada. Finalmente, se espera encontrar una mayor diversidad de géneros tolerantes al disturbio en los arroyos perturbados que en los conservados.

\section{MATERIALES Y MÉTODOS}

Área de estudio: El estudio se realizó en el sector sur del complejo de páramos Rabanal, ubicado entre los Departamentos de Cundinamarca y Boyacá, Colombia (Fig. 1). Las condiciones climáticas del complejo favorecen la oferta hídrica, debido a las diferencias de humedad de las vertientes. La precipitación promedio anual varía entre 650 y $950 \mathrm{~mm}$. El régimen de distribución de las lluvias es bimodal, con un pico entre marzo y julio y otro de 




Fig. 1. Ubicación geográfica de la zona de estudio. La microcuenca Quebrada Honda (1-Minas, 2-Cuacuanal, 3-Acueducto 1) corresponde al sistema perturbado y la microcuenca Lenguazaque al sistema conservado (4-Carrizal, 5-Alisal y 6-Peñalisa). Los números corresponden a los arroyos.

Fig. 1. Study area whithin the Paramo Rabanal. The three sites at Quebrada Honda subwatershed correspond to the impaired system (1-Minas, 2-Cuacuanal, 3-Acueducto 1), and the other three at Lenguazaque correspond to the conserved one (4-Carrizal, 5-Alisal y 6-Peñalisa). Numbers represent sites at each subwatershed.

octubre a noviembre (Morales et al., 2007). El páramo Rabanal se clasifica como semihúmedo a seco de acuerdo con la clasificación climática Caldas-Lang, que se basa en la variación altitudinal de la temperatura y la efectividad de la precipitación (Gutiérrez, 1991). La estructura del paisaje corresponde a un mosaico en el que predomina la vegetación herbácea, tanto en el páramo natural, como en las zonas afectadas por la actividad humana (Morales et al., 2007).

Se seleccionaron seis arroyos en dos microcuencas con diferentes estados de conservación (Fig. 1, Cuadro 1). En la microcuenca Lenguazaque (conservada) se estudiaron dos arroyos al interior de la reserva Páramo de Rabanal y uno en el límite este, donde aún persisten fragmentos de ecosistemas naturales de la región paramuna. Este sitio se localizada en el sector nororiental del municipio de Lenguazaque, y cubre un área aproximada de 8200 ha y presenta altitudes que van desde los 2500 hasta los $3400 \mathrm{msnm}$. En la microcuenca Quebrada Honda (perturbada) se estudiaron también tres arroyos que presentan diversas actividades humanas, tales como minería (explotación de carbón), cultivos y casas aledañas. El Páramo de Rabanal inicia sobre los 3 430msnm y tiene un área total de 5200 ha (Morales et al., 2007).

Diseño de muestreo: En cada uno de los seis arroyos se realizaron cuatro muestreos en total, dos en septiembre de 2008 y mayo de 2009 correspondientes a época lluviosa y otros dos durante noviembre de 2008 y febrero de 
CUADRO 1

Caracterización ambiental de los seis arroyos estudiados en el Páramo Rabanal, Boyacá y Cundinamarca, Colombia

TABLE 1

Environmental characterization of the six streams studied at the Páramo Rabanal, Boyacá y Cundinamarca, Colombia

\begin{tabular}{lcccccc}
\multirow{2}{*}{ Parámetros } & \multicolumn{3}{c}{ Microcuenca conservada } & \multicolumn{3}{c}{ Microcuenca perturbada } \\
& Carrizal & Alisal & Peñalisa & Acueducto & Cuacuanal & Minas \\
Coordenadas & $5^{\circ} 21^{\prime} \mathrm{N}$ & $5^{\circ} 21^{\prime} \mathrm{N}$ & $5^{\circ} 20^{\prime} \mathrm{N}$ & $5^{\circ} 24^{\prime} \mathrm{N}$ & $5^{\circ} 24^{\prime} \mathrm{N}$ & $5^{\circ} 24^{\prime} \mathrm{N}$ \\
& $73^{\circ} 36^{\prime} \mathrm{W}$ & $73^{\circ} 36^{\prime} \mathrm{W}$ & $73^{\circ} 36^{\prime} \mathrm{W}$ & $73^{\circ} 37^{\prime} \mathrm{W}$ & $73^{\circ} 38^{\prime} \mathrm{W}$ & $73^{\circ} 39^{\prime} \mathrm{W}$ \\
Altitud $(\mathrm{msnm})$ & 3310 & 3277 & 3052 & 3303 & 3062 & 2926 \\
Orden & $1^{\circ}$ & $1^{\circ}$ & $2^{\circ}$ & $1^{\circ}$ & $3^{\circ}$ & $3^{\circ}$ \\
Microhábitats & $1,2,4,5,6,7$ & $1,2,4,5,6,7$ & $1,2,3,4,5,6,7,8$ & $1,3,4,5,6,7,8$ & $1,3,4,5,6,7$ & $1,3,4,6,7$ \\
Ancho $(\mathrm{m})$ & $1.51 \pm 0.66$ & $1.51 \pm 0.62$ & $2.98 \pm 1.11$ & $5.80 \pm 2.68$ & $1.67 \pm 0.82$ & $3.38 \pm 0.98$ \\
Profundidad $(\mathrm{m})$ & $0.32 \pm 0.09$ & $0.31 \pm 0.24$ & $0.38 \pm 0.11$ & $0.45 \pm 0.16$ & $0.39 \pm 0.20$ & $0.28 \pm 0.11$ \\
$\mathrm{O}_{2}$ Disuelto $(\mathrm{mg} / \mathrm{l})$ & $8.50 \pm 1.41$ & $7.58 \pm 0.59$ & $7.10 \pm 0.44$ & $6.42 \pm 0.94$ & $6.30 \pm 0.54$ & $6.52 \pm 2.61$ \\
Conductividad $(\mu \mathrm{s})$ & $5.60 \pm 2.07$ & $18.88 \pm 7.43$ & $18.71 \pm 14.43$ & $1.21 \pm 1.51$ & $19.00 \pm 9.58$ & $30.75 \pm 22.59$ \\
SDT* $(\mathrm{ppm})$ & $5.67 \pm 6.09$ & $9.29 \pm 3.73$ & $6.14 \pm 1.46$ & $0.33 \pm 0.47$ & $9.71 \pm 4.62$ & $25.43 \pm 37.15$ \\
Temperatura $\left({ }^{\circ} \mathrm{C}\right)$ & $10.77 \pm 0.86$ & $10.96 \pm 0.94$ & $12.26 \pm 0.46$ & $12.66 \pm 1.24$ & $13.66 \pm 1.00$ & $13.55 \pm 1.49$ \\
$\mathrm{pH}$ & $7.30 \pm 0.31$ & $7.54 \pm 0.71$ & $7.37 \pm 0.42$ & $5.46 \pm 0.17$ & $6.99 \pm 0.18$ & $5.96 \pm 0.86$ \\
\hline
\end{tabular}

*Sólidos Disueltos Totales.

Los números en microhábitats corresponden a: 1: pequeñas zonas de cascada, 2: gravilla, 3: hojarasca, 4: musgos en corrientes lentas, 5: musgos en corrientes rápidas, 6: rocas en corrientes lentas, 7: rocas en corrientes rápidas, 8: zonas de salpicadura.

2009 durante la época seca. En cada arroyo se seleccionó un transecto que incluyera todos los posibles microhábitats en los que habitan las larvas de tricópteros, como piedras en corrientes lentas, rápidas, musgos y hojarasca (Cuadro 1) (Rincón, 1996). La longitud del transecto, que fue diez veces el ancho del cauce, y el número de microhábitats variaron en cada arroyo dependiendo de la estructura de cada sitio. Características como el ancho del cauce, aportes alóctonos, presencia de musgos, velocidad de la corriente y pendiente permiten que haya diferentes sustratos para que las larvas se establezcan, por lo que el número de microhábitats por arroyo fue diferente. Una vez elegido el transecto y los microhábitats, los especímenes se recolectaron con una red tipo D de $30.5 \mathrm{~cm}$ de diámetro con una abertura de malla de $150 \mu \mathrm{m}$. Se realizaron tres redadas a lo largo del transecto por cada microhábitat muestreado. Cada redada consistió en colocar la red a contracorriente en el microhábitat a muestrear, luego se procedió hacer un barrido manual, y finalmente la inspección en campo sobre los sustratos duros como rocas y en el caso de hojarasca y musgos se recolectó material para revisión el laboratorio.

Durante cada muestreo en cada transecto de los arroyos se midió la profundidad y la zona de inundación con una vara graduada de $1 \mathrm{~m}$. El oxígeno disuelto fue medido usando el método Winkler, mientras que la temperatura del agua, sólidos disueltos totales, conductividad y $\mathrm{pH}$ con un equipo multiparamétrico (MILWAUKEE Modelo Mi805). Con el fin de obtener datos más precisos se tomaron tres medidas para cada parámetro mencionado: una en la parte alta, otra en la parte media y otra en la parte baja de cada transecto.

En el laboratorio los tricópteros se determinaron a nivel de género con la ayuda de claves taxonómicas especializadas (Wiggins, 1996; Posada \& Roldán, 2003) y la posterior corroboración con un especialista. Los especímenes recolectados fueron preservados en frascos con etanol al 70\% y se depositaron en la 
colección del Instituto de Investigaciones Biológicas Alexander von Humboldt, sede Villa de Leyva, Boyacá (Colombia) con la base de datos correspondiente.

Análisis de datos: Para analizar los patrones de las comunidades de tricópteros entre las microcuencas se usaron curvas de dominanciadiversidad (Feinsinger, 2001). Para comparar estadísticamente la riqueza y abundancia de los géneros de arroyos y microcuencas se emplearon curvas de rarefacción basadas en individuos y sus intervalos de confianza; cada redada fue considerada una unidad de muestreo. Se determinó si la diversidad observada de la comunidad más pequeña se encuentra dentro del 95\% de los límites de confianza de la curva de rarefacción de la comunidad más grande. La comparación fue hecha en el punto en el que el nivel de abundancia de la comunidad grande alcanza el nivel de la más pequeña (Gotelli \& Colwell, 2001). Para predecir la riqueza de géneros y evaluar la eficiencia del muestreo en cada uno de los arroyos y en cada microcuenca se utilizaron los estimadores no-paramétricos Chao1 y Chao2, (Colwell \& Coddington, 1994) con la ayuda del programa EstimateS v. 8.2 (Colwell, 2005). Estos estimadores han sido recomendados para el estudio de la comunidad de macroinvertebrados de sistemas acuáticos tropicales (Melo \& Froehlich, 2001). Se realizaron 100 aleatorizaciones para cada curva (Colwell, 2005).

Para determinar la contribución relativa de los componentes alfa y beta a la diversidad gamma se empleó el análisis de partición multiplicativa de la diversidad (Jost, 2007; Jost et al., 2010; Tuomisto, 2010). Para calcular el aporte de cada componente se utilizaron dos medidas de diversidad: la riqueza (i.e., géneros) y la diversidad de Shannon, expresados como números efectivos (medidas de diversidad verdadera), de orden $q=0\left({ }^{0} D\right)$ y $q=1\left({ }^{1} D\right)$ respectivamente (Jost, 2007; Jost et al., 2010; Rös, Escobar \& Halffter, 2012):

$$
\begin{gathered}
{ }^{0} D_{\gamma}=S \\
{ }^{0} D_{\alpha}=(1 / N)\left(S_{1}+S_{2}+\ldots+S_{j}\right) \\
{ }^{0} D_{\beta}={ }^{0} D_{\alpha} /{ }^{0} D_{\gamma}
\end{gathered}
$$

${ }^{0} D_{\gamma}$ corresponde al número total de géneros encontrados por microcuenca. $S_{j}$ es el número de géneros por unidad de muestreo y $N$ es el número de unidades de muestreo.

$$
\begin{gathered}
{ }^{1} D_{\gamma}=\exp \left[-\sum_{n=1}^{s}\left(p_{i} \ln p_{i}\right)\right] \\
{ }^{1} D_{\gamma}=\exp \left[-w_{1} \sum_{n=1}^{s}\left(p_{i l} \ln p_{i I}\right)+w_{2} \sum_{n=1}^{s}\left(p_{i 2} \ln p_{i 2}\right) \ldots\right] \\
w_{j}=\text { ind }_{j} / \text { ind } \\
{ }^{1} D_{\beta}={ }^{1} D_{\gamma} /{ }^{1} D_{\alpha}
\end{gathered}
$$

Donde $w$ es el peso estadístico con que contribuye cada unidad de muestreo al total de la diversidad, expresado como el número de individuos en la comunidad $j$ dividido por el número total de individuos en cada microcuenca, en este caso.

La diversidad de orden cero $(q=0)$ es completamente insensible a las abundancias encontradas; por lo tanto, este valor equivale a la riqueza de géneros, en este caso. Los valores de $q$ menores de 1 sobrevaloran las especies raras; cuando $q=1$, todas las especies son incluidas con un peso exactamente proporcional a su abundancia en la comunidad (Hill, 1973; Moreno, Barragán, Pineda \& Pavón; 2011). Los resultados se expresan en unidades que han sido llamadas número efectivo de especies, número de especies equivalentes o especies igualmente comunes (Jost, 2007). Cuando se usan estas medidas de diversidad para calcular la diversidad de beta, se infiere que es baja cuando una determinada comunidad domina en la unidad de paisaje, ya que no hay recambio entre las unidades de muestreo que se tuvieron en cuenta (Jost, 2007).

Para el análisis de los cambios entre arroyos considerando como atributos las abundancias 
de los géneros de cada arroyo, se utilizó un Análisis de Agrupamiento con el programa PC-ORD v5 (McCune \& Mefford, 1999). El dendrograma fue elaborado utilizando como medida de distancia o similitud el índice BrayCurtis y UPGMA (Unweighted Pair Group Method Using Arithmetic Average, por sus siglas en inglés) como algoritmo de agrupamiento. Las abundancias fueron transformadas $(\log [\mathrm{x}+1])$ con el objeto de disminuir el efecto de los géneros con mayor abundancia (Clark \& Warwick, 2001).

\section{RESULTADOS}

Se recolectaron un total de 1934 individuos, pertenecientes a 16 géneros y 9 familias de tricópteros. La curva de rarefacción de la microcuenca perturbada (Quebrada Honda) parece no haber alcanzado la asíntota y sus intervalos de confianza son amplios, lo que sugiere que el número de géneros puede ser mayor, mientras que la curva de rarefacción de la microcuenca conservada (Lenguazaque) se acercó mucho a la asíntota y sus intervalos de confianza fueron más estrechos (Fig. 2).
Sin embargo, no se encontraron diferencias significativas de la riqueza entre ambas microcuencas. La relación entre la abundancia y la dominancia fue diferente entre las microcuencas. En Lenguazaque los géneros dominantes fueron Helicopsyche, Ochrotrichia y Contulma, mientras que para Quebrada Honda fueron Smicridea, Xiphocentron y Ochrotrichia (Fig. 3). La familia Hydroptilidae estuvo representada en ambas microcuencas por cinco géneros; de éstos únicamente el género Hydroptila fue exclusivo de la microcuenca conservada, junto con Triplectides y Oecetis de la familia Leptoceridae. Por otro lado, los géneros Banyallarga y Polycentropus se encontaron únicamente en Quebrada Honda. Tanto a escala de microcuenca como de arroyos los sistemas conservados presentan un mayor número de individuos que los perturbados ( $t$ Student: $\mathrm{p}<0.001$ ) (Cuadro 2).

En Lenguazaque se encontraron 14 géneros y más de 1600 individuos. Las curvas de rarefacción de los arroyos conservados sugieren que la riqueza de géneros de Carrizal y Alisal fue muestreada en su totalidad (Fig. 4). Los intervalos de confianza de las curvas de rarefacción mostraron traslape, lo que sugiere

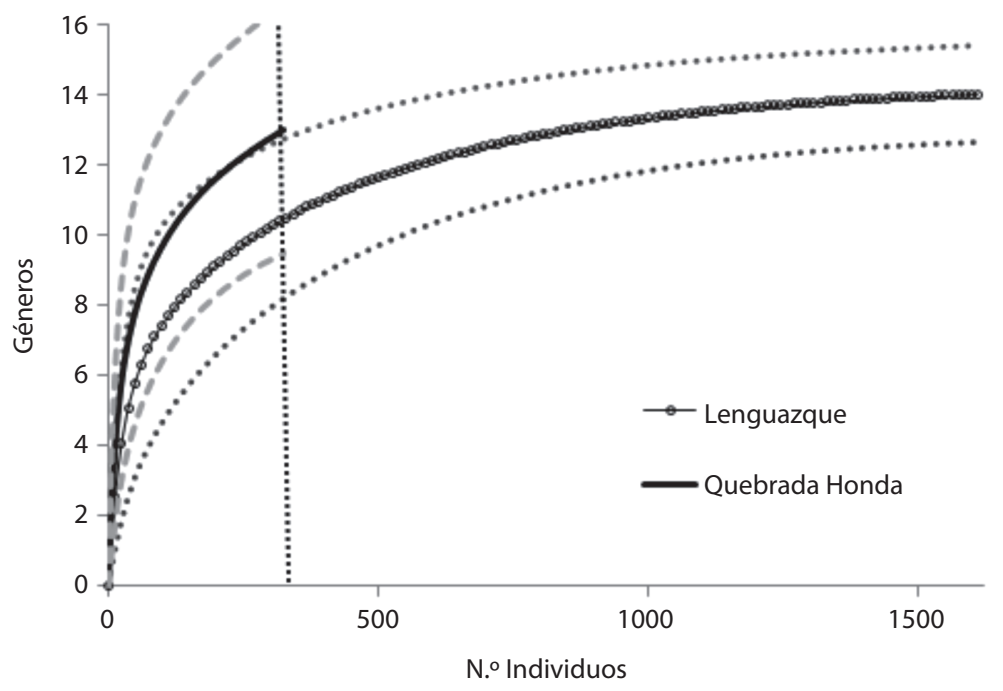

Fig. 2. Curvas de rarefacción de Trichoptera en las microcuencas conservada (Lenguazaque) y perturbada (Quebrada Honda). La línea punteada vertical muestra el punto en el que serán comparadas las microcuencas.

Fig. 2. Rarefaction curves for Trichoptera from Lenguazaque (conserved) and Quebrada Honda (impaired) watersheds. 


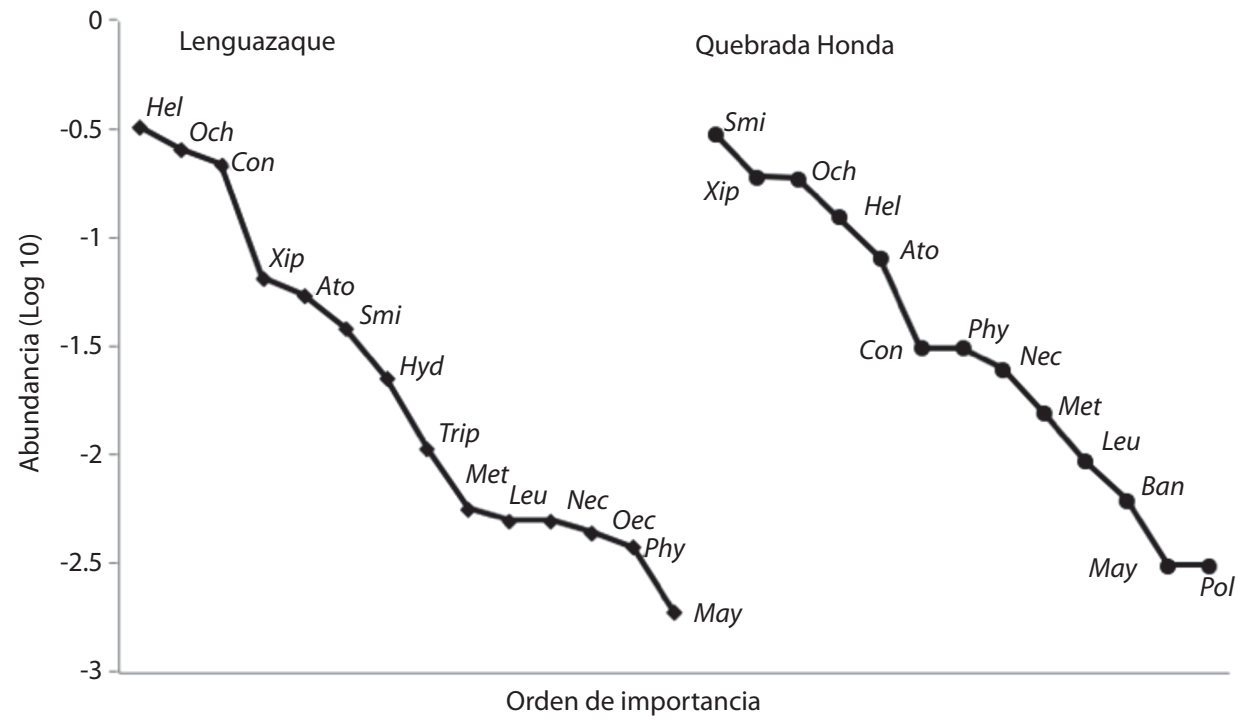

Fig. 3. Gráfico de la dominancia- abundancia para los géneros de Trichoptera encontrados en las microcuencas Lenguazaque (conservada) y Quebrada Honda (perturbada). La abreviatura de cada punto corresponde a las tres primeras letras de cada género que están registradas en el Cuadro 1.

Fig. 3. Dominance- abundance graph of genera of Trichoptera sampled at conserved (Lenguazaque) and impaired (Quebrada Honda) watersheds. Letters in each point represent the abbreviation of each genus in table 1.

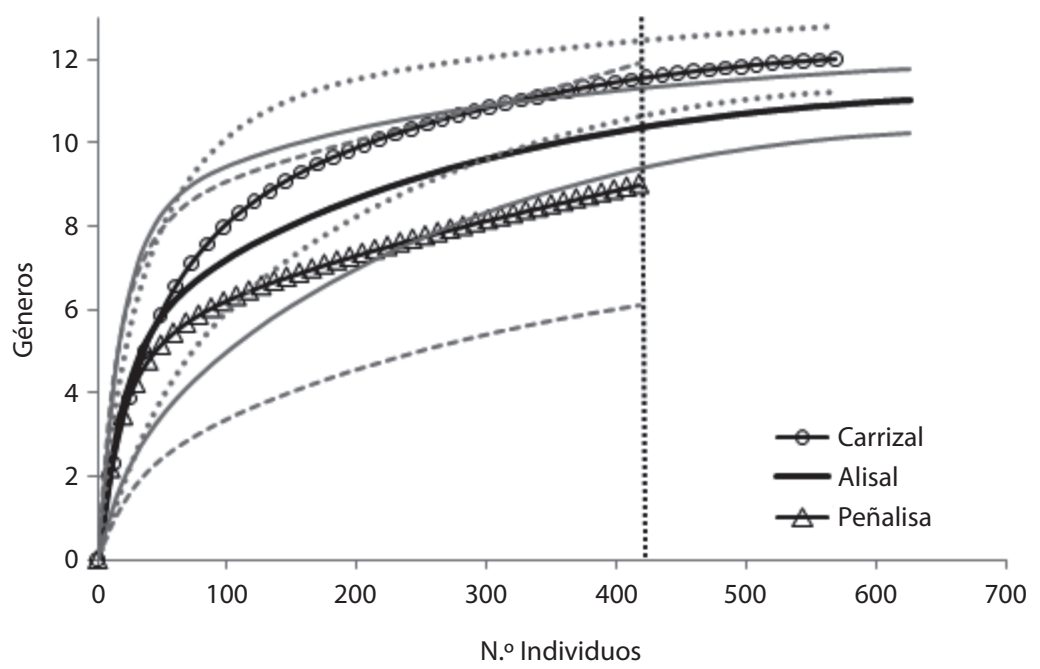

Fig. 4. Curvas de rarefacción del orden Trichoptera en los arroyos de la microcuenca conservada (Lenguazaque). La línea punteada vertical indica el punto en el que son comparados los comunidades de los tres arroyos.

Fig. 4. Rarefaction curves of Trichoptera in the streams of the conserved watershed (Lenguazaque). Vertical dotted line indicates the point where the assemblages from the three streams are compared. 


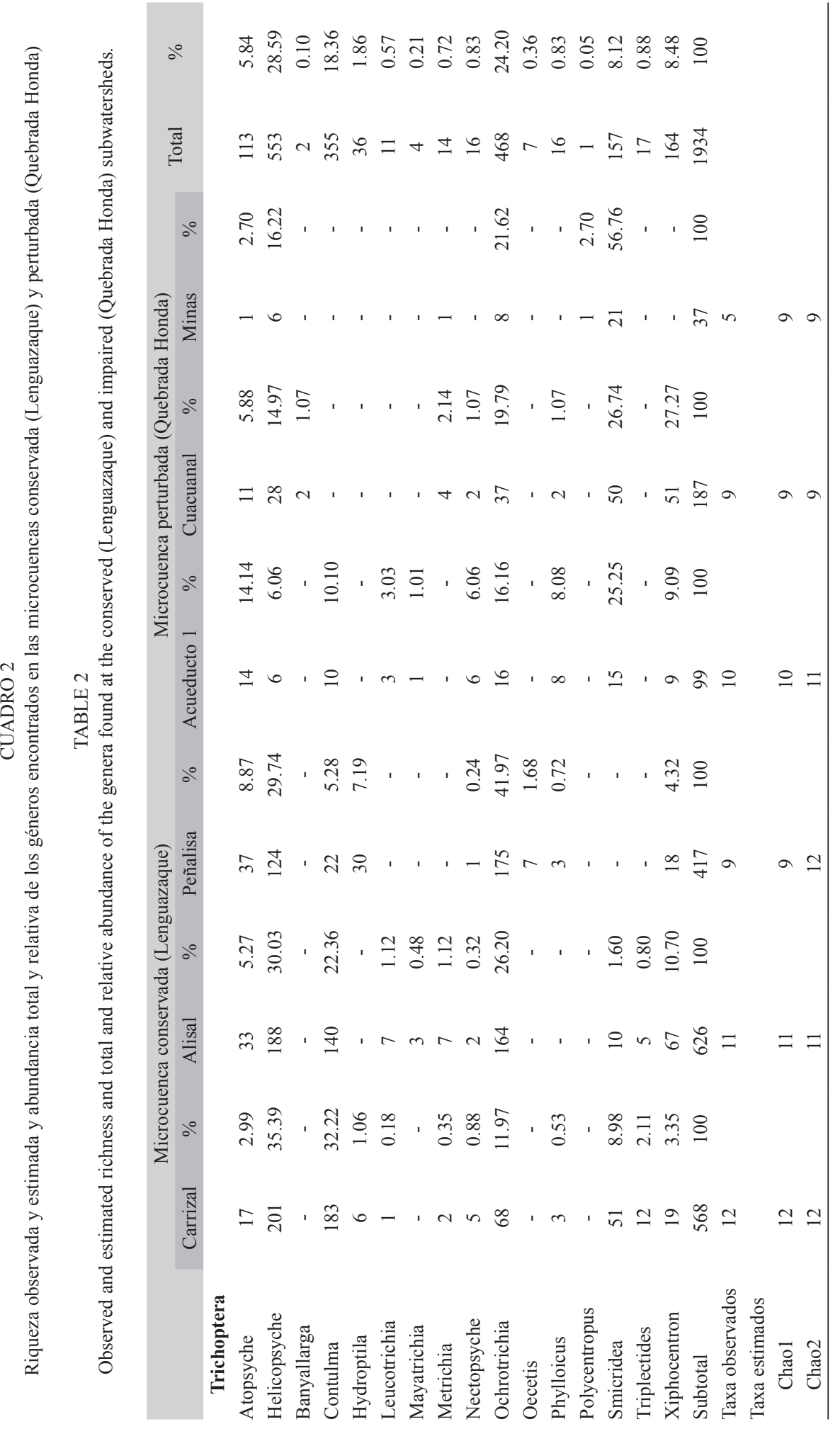


que no hay diferencias significativas en riqueza de géneros entre ellos. Los estimadores Chao 1 y Chao 2 mostraron que el porcentaje de eficiencia de muestreo para los tres arroyos de esta microcuenca estuvo entre $75 \%$ y 100\% (Cuadro 2).

En Quebrada Honda se encontraron 13 géneros y cerca de 300 individuos (Fig. 5). Ninguna de las curvas de rarefacción de los arroyos de Quebrada Honda alcanzaron la asíntota y sus intervalos de confianza son muy amplios. La curva de rarefacción de Minas se traslapa con los intervalos de confianza de Acueducto y Cuacuanal (Fig. 5A), mientras que la de Cuacuanal no se traslapa con los intervalos de confianza de Acueducto, lo que sugiere que Cuacuanal tiene significativamente menos géneros que Acueducto (Fig. 5B). En general, el esfuerzo de muestreo para esta microcuenca parece haber sido adecuado de acuerdo con los estimadores Chao 1 y Chao 2 con porcentajes de eficiencia del $100 \%$, excepto en Minas (56\%).

En la microcuenca conservada los valores de diversidad alfa de la riqueza $\left({ }^{0} D\right)$ y de la diversidad de Shannon $\left({ }^{1} D\right)$ fueron muy similares entre los arroyos que se encuentran dentro de la Reserva del páramo Rabanal (Alisal y Carrizal), mientras que en Peñalisa, que se encuentra en el límite de la reserva, ambas medidas fueron menores (Cuadro 3 ). En cambio, ambos valores de alfa $\left({ }^{0} D\right.$ y $\left.{ }^{l} D\right)$ en la microcuenca perturbada reflejaron un gradiente de diversidad, siendo Acueducto el arroyo que presento los valores mas altos y Minas los menores. El componente gamma para la riqueza y para la diversidad de Shannon fue mayor en la microcuenca conservada que en la perturbada por un género. Sin embargo, el valor alfa promedio para la riqueza de la microcuenca conservada fuen mayor por dos géneros en comparación con la perturbada, mientras que la diversidad de Shannon fue prácticamente un género mayor en la cuenca perturbada que en la conservada. Los valores de diversidad beta tanto para la riqueza como para la diversidad de Shannon mostraron que practicamente hay una comunidad establecido por microcuenca, ya que sus valores fueron cercanos a 1. Sin embargo, en la microcuenca perturbada el valor de beta obtenido con la riqueza mostró que hay más diferencias entre

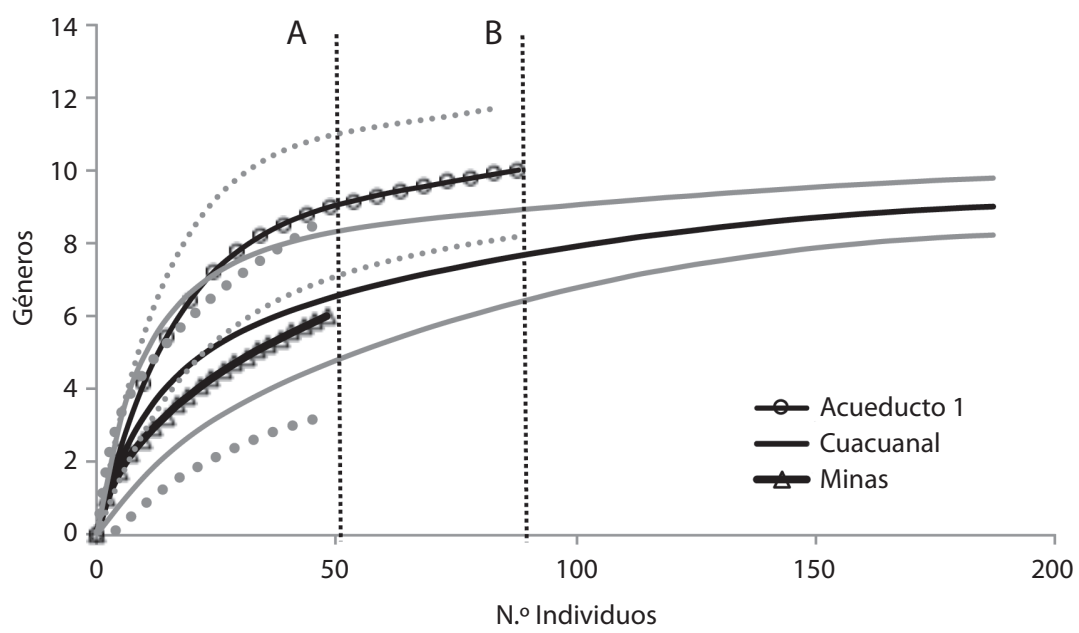

Fig. 5. Curvas de rarefacción de Trichoptera en los arroyos de la microcuenca perturbada (Quebrada Honda). La línea punteada indica el punto en el que se compararon los comunidades. A Comparación de Minas con Cuacuanal y Acueducto, B Comparación de Cuacuanal y Acueducto.

Fig. 5. Rarefaction curves of Trichoptera in the streams of the impaired watershed (Quebrada Honda). Vertical dotted line indicates the point where the assemblages from the three streams are compared. Comparison of Minas with Cuacuanal and Acueducto (A), comparison between Cuacuanal and Acueducto (B). 
CUADRO 3

Partición multiplicativa de las microcuencas Lenguazaque y Quebrada Honda

TABLE 3

Multiplicative diversity partition for conserved and impaired subwatersheds

\begin{tabular}{lcccccc} 
& \multicolumn{3}{c}{ Lenguazaque (Conservada) } & \multicolumn{3}{c}{ Quebrada Honda (Perturbada) } \\
& Alisal & Carrizal & Pefialisa & Acueducto & Cuacuanal & Minas \\
Componentes de la diversidad & ${ }^{0} \mathrm{D} /{ }^{1} \mathrm{D}$ & ${ }^{0} \mathrm{D} /{ }^{1} \mathrm{D}$ & ${ }^{0} \mathrm{D} /{ }^{1} \mathrm{D}$ & ${ }^{0} \mathrm{D} /{ }^{1} \mathrm{D}$ & ${ }^{0} \mathrm{D} /{ }^{1} \mathrm{D}$ & ${ }^{0} \mathrm{D} /{ }^{1} \mathrm{D}$ \\
Diversidad $\alpha$ (Arroyos) & $12 / 5.21$ & $11 / 5.42$ & $9 / 4.65$ & $10 / 8.4$ & $9 / 5.5$ & $6 / 2.95$ \\
Diversidad $\gamma$ (Microcuencas) & & $14 / 5.88$ & & & $13 / 6.9$ & \\
Diversidad $\alpha$ (Microcuencas) & & $10.6 / 4.96$ & & & \\
Diversidad $\beta$ (Microcuencas) & & $1.32 / 1.19$ & & & $1.56 / 1.22$ \\
\hline
\end{tabular}

Los componentes de diversidad corresponden a la diversidad gamma $(\gamma)$, diversidad alfa promedio $(\alpha)$ y diversidad beta $(\beta)$ usando como medidas de diversidad riqueza $\left({ }^{0} D\right)$ e índice de Shannon $\left({ }^{1} D\right)$.

The components of diversity are: gamma diversity $(\gamma)$, mean alpha diversity $(\alpha)$, and beta diversity $(\beta)$ for richness $\left({ }^{0} D\right)$ and Shannon diversity $\left({ }^{1} D\right)$.

los arroyos, probablemente debido a que en Minas la diversidad alfa para ${ }^{0} D$ y ${ }^{1} D$ fue más baja (entre 5 y 4 géneros menos, respectivamente) en comparación con Acueducto.

Al comparar la similitud entre los arroyos considerando los géneros como atributos de cada uno de ellos, se observó que éstos se agrupan de acuerdo al estado de conservación de la microcuenca (Fig. 6). El agrupamiento mostró una mayor semejanza de arroyos de la microcuenca conservada, siendo Carrizal, Alisal y Peñalisa más similares (entre 80 a $100 \%$ de similitud), mientras que los arroyos de la microcuenca perturbada tienen una composición similar entre 37 y $58 \%$. Minas, que fue identificado como el sitio más influenciado por la industria minera fue el más lejano en el dendograma.

De las variables ambientales medidas se observaron diferencias entre los arroyos de las microcuencas en el oxígeno disuelto, la temperatura y el $\mathrm{pH}$ (Cuadro 1). Los valores de oxígeno disuelto oscilaron entre 7.1 y $8.5 \mathrm{mg} / \mathrm{L}$ en los arroyos de la microcuenca conservada, mientras que para la perturbada varió entre 6.3 y $7.1 \mathrm{mg} / \mathrm{L}$. La temperatura, que en alta montaña de las zonas tropicales suele ser fría, fue de 10 a $12.2^{\circ} \mathrm{C}$ en los arroyos de la microcuenca perturbada y de 12.2 a $13.5^{\circ} \mathrm{C}$ en los de la microcuenca perturbada. En cuanto al $\mathrm{pH}$ se observaron valores neutros en los sitios conservados (7.3 a 7.5) mientras que en los perturbados fueron más ácidos (entre 5.4 y 6.9).

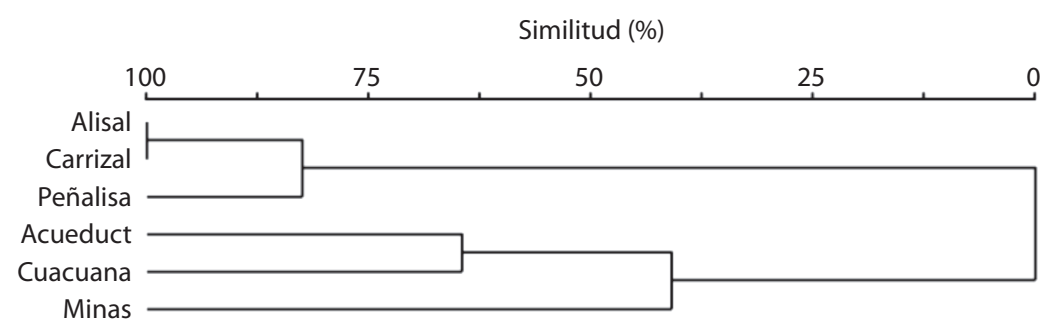

Fig. 6. Dendograma de similitud entre arroyos a partir del Índice de Sorënsen cuantitativo para Trichoptera usando UPGMA como método de amalgamiento.

Fig. 6. Cluster analysis for the streams, based on Sorënsen quantitative index for Trichoptera, with UPGMA as linking method. 


\section{DISCUSIÓN}

Nuestro estudio determinó que la comunidad de tricópteros se vio afectado de acuerdo al gradiente de impacto en cada una de las microcuenca estudiadas. Aunque la riqueza de géneros difirió muy poco entre las microcuencas, la composición varió entre ellas. Los géneros Helicopsyche y Ochrotrichia estuvieron presentes en los arroyos de ambas microcuencas, pero fueron más abundantes en la conservada. Estos géneros han sido reportados anteriormente para la región y en cotas altitudinales que van de 2600 a $3550 \mathrm{msnm}$, en sitios de bosque alto andino y páramo (La Rotta, 1989; Rincón, 1996; Posada, Abril \& Parra, 2008). Helicopsyche vive sobre rocas en áreas de corriente moderada donde raspan algas; algunas especies de este género pueden tolerar considerables niveles de contaminación orgánica (Springer, 2010). El género Ochrotrichia se encuentra dividido en dos subgéneros; los individuos encontrados en este estudio probablemente pertenecen al subgénero Metrichia, común en zonas de corriente o sobre rocas húmedas de cuerpos de agua de alta montaña sobre los Andes (Flint, 1983). La distribución restringida de este subgénero lo hace particularmente vulnerable; la disminución en la abundancia en los arroyos perturbados podría sugerir un efecto negativo sobre este taxón.

Contulma fue encontrado en todos los arroyos de la microcuenca conservada y en Acueducto de la microcuenca perturbada, pero con menor abundancia. Estos insectos frecuentan zonas de cascadas, riachuelos y pequeñas quebradas tanto en áreas de bosque alto andino como en páramos. De este género se conocen 18 especies de países del norte de los Andes, todas por encima de los $2000 \mathrm{msnm}$. Los especímenes de este género que son escasos en las recolectas, parecen mostrar un alto endemismo local (Holzenthal \& Ríos, 2012). Se esperaba encontrar este género debido a que es frecuente en ecosistemas andinos con las características de los sitios estudiados, así que su ausencia en los arroyos Cuacuanal y Minas de la microcuenca perturbada puede que sea una respuesta a los efectos de las actividades antrópicas de la zona.

Los géneros de Leptoceridae y Calamoceratidae encontrados y que presentaron las menores abundancias se caracterizan porque construyen refugios con material vegetal como hojarasca y pequeños trozos de troncos dentro del agua (Wiggins, 1996). Banyallarga y Phylloicus (Calamoceratidae) se encuentran con frecuencia en áreas de corriente lenta especialmente durante épocas secas (Posada \& Roldan, 2003). Para la microcuenca perturbada (arroyo Cuacuanal) se registraron solo dos especímenes de Banyallarga, un género raro en las recolectas, y de distribución restringida a Centroamérica y el norte de Suramérica (Prather, 2004). Phylloicus se encontró en los arroyos Acueducto 1 y Cuacuanal. Ninguno de los dos géneros mencionados estuvo presente en Minas. Esto probablemente refleje la condición ecológica de los arroyos de la microcuenca perturbada, ya que estos géneros pueden sobrevivir en lugares con cierto nivel de contaminación (Roldán, 2003) como los de Cuacuanal y Acueducto, pero no tan extremo como el de Minas. Smicridea, el género más abundante del sistema contaminado, es un género muy común y ampliamente distribuido con cierto grado de tolerancia a los sistemas de este tipo (Roldán, 2003).

La diversidad local de los arroyos, medida a través de la riqueza y la diversidad de Shannon, mostró mayores diferencias entre los arroyos de la microcuenca perturbada, que podrían estar afectados por las actividades antrópicas de diferente forma que los de la microcuenca conservada. En el caso de Acueducto, existe modificación del sistema natural como ampliación y represamiento del cauce natural, introducción de especies vegetales y presencia de cultivos (obs pers.). A pesar de estos cambios, la diversidad de las comunidades de tricópteros es similar a la de los de los arroyos de la cuenca conservada; inclusive, la diversidad de Shannon fue la mayor de todos los sitios de estudio. Cuacuanal presenta una situación similar, con valores de riqueza y diversidad de Shannon más cercanos a los de 
los arroyos de la microcuenca conservada. El gradiente de diversidad que se observa en los arroyos de la microcuenca perturbada sugiere que la actividad minera es la que más afecta las comunidades de tricópteros, ya que es la principal actividad en la zona. Aunque las modificaciones estructurales en Acueducto y Cuacuanal ofrecen nuevos microhábitats que son aprovechados por los tricópteros, puede que algunas especies ó géneros (como Contulma, Ochrotrichia o Hydroptila, que disminuyen su abundancia o no están presentes en los arroyos de la microcuenca perturbada) no toleren estos cambios. El hecho de que la magnitud de recambio no haya sido tan diferente entre las microcuencas, no significa que no se esté perdiendo diversidad y no se haya modificado la estructura de la comunidad, esto último confirmado también por el análisis de similitud, que mostró que los arroyos se separaron claramente entre microcuencas, considerando como atributos los géneros de los tricópteros. El hecho de que los valores de diversidad beta entre microcuencas para la diversidad de Shannon fueron menores que los calculados para la riqueza, indica que la variación en la diversidad entre las microcuencas se debió a los géneros raros o poco abundantes (véase Jost, 2006).

En cuanto a las variables in situ, al comparar la temperatura, el oxígeno disuelto y el $\mathrm{pH}$ resultaron diferentes entre los arroyos de las microcuencas. En los arroyos de la microcuenca perturbada la temperatura mostró un incremento en su valor promedio con respecto a los arroyos de la conservada, mientras que el oxígeno disuelto que es considerado un indicador importante de la calidad del agua y que normalmente osila entre los 7 y $8 \mathrm{mg} / \mathrm{L}$, disminuyó y según los valores de $\mathrm{pH}$ sus aguas son más ácidas. A pesar de la complejidad climatológica de los páramos y los pocos estudios realizados en este ecosistema se observa que al comparar con otros trabajos los sistemas de la microcuenca perturbada tienen valores más cercanos a los de bosques alto andinos que a los de páramo. Díaz y Rivera (2004) por ejemplo, caracterizaron algunos ríos pequeños de la zona alto andina en Cundinamarca (Colombia), ubicados entre los 2617 y $3107 \mathrm{msnm}$, en una zona de baja influencia antrópica. En este estudio se encontraron valores promedio de temperatura de $14.8^{\circ} \mathrm{C}, \mathrm{pH}$ de 5.8 y oxígeno disuelto de $6,86 \mathrm{mg} / \mathrm{L}$, que resultan cercanos a los valores en la microcuenca perturbada. En contraste Jacobsen, Schultz \& Encalada (1997) en un estudio sobre la influencia de la temperatura sobre la comunidad de macroinvertebrados de diferentes regiones incluida la zona de páramo, encontraron valores de temperatura de $9.8^{\circ} \mathrm{C}$ y 7.39 de $\mathrm{pH}$, que son similares a los encontrados en este estudio para la microcuenca conservada.

Para ser más concluyentes y entender mejor la relación de los tricópteros con las variables fisicoquímicas, se recomienda que estudios futuros incluyan en sus diseños análisis que reflejen el efecto de los contaminantes como los de las industrias presentes en la región, las concentraciones de nitrato y ortofosfato aportadas por la agricultura, así como las de sulfato por la actividad minera, entre otros. Aún así, el presente estudio provee una idea general del grado de influencia que tienen las condiciones ambientales en las que viven los organismos sobre la composición y abundancia de los mismos en ecosistemas lóticos, y provee una referencia para futuros estudios.

El orden Trichoptera representa una gran diversidad de formas de vida en los páramos, que a pesar de considerarse biomas poco diversos, el alto endemismo los hace ecosistemas únicos. Esta diversidad se ve reflejada no solo en el número de géneros y especies, sino también en sus hábitos alimentarios, construcción de refugios, adaptaciones a condiciones extremas y en las funciones que cumplen dentro del ecosistema (Mackay \& Wiggins, 1979; Wiggins, 2004; Springer, 2010). Este estudio mostró que la diversidad de tricópteros es diferente entre el sistema conservado y perturbado y que esto podría deberse a las actividades antrópicas. Se sugiere realizar estudios que permitan establecer en qué forma y grado las diferentes actividades llevadas a cabo en el páramo afectan este y otras comunidades de insectos acuáticos, dando prioridad a la biología y taxonomía de los géneros/especies característicos 
de estas zonas, que podrían estar en alto riesgo. Esta información sería útil para tomar medidas para su conservación y que han sido efectivas en el sector de la microcuenca Lenguazque, ya que han cumplido con el objetivo de proteger la diversidad paramuna de tricópteros allí presente. De no proteger estos ecosistemas los géneros y especies endémicas de esta importante zona andina pobablemente sean las que están en mayor riesgo.

\section{AGRADECIMIENTOS}

A Federico Escobar Sarria, Juan Manuel Pech y José Antonio Gómez del Instituto de Ecología A.C. (México) por su aporte en el análisis estadístico y revisiones. A María Eugenia Rincón (Q.E.P.D.) de la Universidad Pedagógica Nacional (Colombia) por la determinación y corroboración de los especímenes. A Beatriz Bolívar Cimé por el diseño del mapa. A las colaboradoras en la fase de campo Pilar Latorre B., Mónica Torres, Rosa María Bohórquez y Angela A. Camargo. Este estudio fue financiado a través de una beca de CODESAN, en el marco del Proyecto Páramo Andino, con el apoyo del Instituto de investigaciones biológicas Alexander von Humboldt.

\section{RESUMEN}

Los tricópteros son considerados un componente biótico importante y diverso de los ecosistemas acuáticos continentales. Este estudio tuvo como objetivo evaluar la comunidad del orden Trichoptera en dos microcuencas con diferente grado de perturbación. Se realizaron cuatro campañas de muestreo en dos microcuencas de la Cordillera Oriental de los Andes colombianos. Se realizaron análisis mediante curvas de rarefacción, el índice de Bray Curtis y partición de la diversidad, usando como medidas la riqueza y la diversidad de Shannon. Se encontró que, aunque la riqueza fue similar entre las dos microcuencas, la abundancia siempre fue mayor en los arroyos de la microcuenca conservada. En cada microcuenca dominaron géneros diferentes, excepto Ochrotrichia que fue abundante en todos los sitios. La diversidad alfa entre los arroyos de la microcuenca conservada fue similar, mientras que en la perturbada se observó una reducción de la diversidad asociada a un gradiente de perturbación de los arroyos. La diversidad beta $\left({ }^{0} D_{\beta}\right.$ y $\left.{ }^{l} D_{\beta}\right)$ entre microcuencas y entre arroyos conservados fue similar, mientras que en los perturbados también mostró un gradiente similar al de la diversidad alfa. El análisis de similitud agrupó los arroyos según su estado de conservación. Las diferencias encontradas en los atributos de los comunidades de tricópteros confirman que su uso es adecuado para conocer el estado de conservación de los sistemas que habita.

Palabras clave: zonas altoandinas, insectos acuáticos, diversidad verdadera, curvas de rarefacción, conservación.

\section{REFERENCIAS}

Bispo, C., \& Leandro, G. O. (2007). Diversity and structure of Ephemeroptera, Plecoptera and Trichoptera (Insecta) assemblages from riffles in mountain streams of central Brazil. Revista Brasilera de Zoología, 24(2), 283-293.

Clarke, K. R., \& Warwick, R. M. (2001). Measures of similarity of species abundance/ biomass between samples. In Change in marine communities: an approach to statistical analysis and interpretation. PRIMER-E Ltd.

Cleef, A. (1978). Caracteristic of neotropical paramo vegettion and it subantartic relations. In C. Troll \& W. Lauer (Eds.). Geological relations beteween the soutrn temperate zone and the tropical mountains (pp. 365 -390). Alemania: Franz estaineir werlag GMBH.

Colwell, R. K. (2005). EstimateS: Statistical estimation of species richness and shared species from samples. Version 7.5. User's Guide and application. (Recuperado de http://purl.oclc.org/estimates)

Colwell, R. K., \& Coddington, J. A. (1994). Estimating terrestrial biodiversity through extrapolation. Philosophical Transactions of the Royal Society of London. Series B: Biological Sciences, 345, 101-118.

Cuatrecasas, J. (1989). Frailejonal, típico cuadro de la vida vegetal en los páramos andinos. Revista Academia Colombiana de Ciencias Exactas, 7(28), 457-461.

Díaz, C., \& Rivera, C. A. (2004). Diatomeas de pequeños ríos andinos y su utilización como indicadoras de condiciones ambientales. Caldasia, 26(2), 381-394.

Feinsinger, P. (2001).Designing field studies for biodiversity conservation. Washington, DC: Island Press.

Flint, O. (1983). Studies or Neotropical caddisflies, XXXIII: new species from Austral South America (Trichoptera). Smithsonian Contributions to Zoology, 377.

Gotelli, N. J., \& Colwell, R. (2001). Quantifying biodiversity: Procedures and pitfalls in the measurement and comparison of species richness. Ecology letters, 4, 379-391.

Gutiérrez, J. H. (1991). Clasificaciones climáticas. Bogotá. Colombia: Instituto colombiano de hidrología, meteorología y adecuación de tierras HIMAT. 
Hill, M. O. 1973. Diversity and evenness: a unifying notation and its consequences. Ecology, 54, 427-432.

Holzenthal, R., \& Ríos, B. (2012). Contulma paluguillensis /Trichoptera: (Anomalopsychidae), a new caddisfly from the high Andes of Ecuador, and its natural history. Freshwater Science, 31(2), 442-450.

Holzenthal, R. W., \& Flint, O. S. (1995). Studies of Neotropical caddisflies, LI: Systematics of the Neotropical caddisfly genus Contulma (Trichoptera: Anomalopsychidae). Smithsonian Contributions to Zoology, 575, 1-59.

Jacobsen, D., Schultz, R. \& Encalada A. (1997) Structure and diversity of stream invertebrate assemblages: the influence of temperature with altitude and latitude. Freshwater Biology, 38, 247-261.

Jost, L. (2006). Entropy and diversity. Oikos, 113, 363-375.

Jost, L. (2007). Partitioning diversity into independent alpha and beta components. Ecology, 88, 2427-2439.

Jost, L., DeVries, P., Walla, T., Greeney, H., Chao, A. \& Ricotta, C. (2010). Partitioning diversity for conservation analyses. Diversity and Distributions, 16, 65-76.

La Rotta, L. (1989). Faunistich-Autokolosch Untersuchung der Tricopteren des Santuario de Fauna y Flora de Iguaque (Boyacá, Kouloumbien), (Tesis de Doctorado). Institut fur Allgemeine und speziele zoologie justus-liegig, Gieben.

Mackay, R., \& Wiggins G. (1979). Ecological diversity in Trichoptera. Annual reviews of Entomology, 24, 185-208.

McCune, B., \& Mefford, M. J. (1999). PC-ORD. Multivariate Analysis of Ecological Data. Version 5.0 MjM Software. Gleneden Beach, Oregon, U.S.A.

Melo, A., \& Froehlich, C. (2001). Evaluation of methods for estimating macroinvertebrate species richness using individual stones in tropical streams. Freshwater Biology, 47, 711-721.

Morales, M., Otero, J., Van der Hammen, T., Torres, A., Cadena, C., Pedraza, C., Rodríguez, N., Franco, C., Betancouth, J. C., Olaya, E., Posada, E., \& Cárdenas, L. (2007). Atlas de páramos de Colombia. Bogotá, D.C.: Instituto de Investigación $m$ de Recursos Biológicos Alexander von Humboldt.
Moreno, C. E., Barragán, F., Pineda E., \& Pavón, N. (2011). Reanálisis de la diversidad alfa: alternativas para interpretar y comparar información sobre comunidades ecológicas. Revista mexicana de biodiversidad, 82, 1249-1261.

Posada, J. A., Abril, G. \& Parra, L. N. (2008). Diversidad de los macroinvertebrados acuáticos del páramo de Frontino (Antioquia, Colombia). Caldasia, 30(2), 441-455.

Posada, A., \& Roldán, G. (2003). Clave ilustrada y diversidad de las larvas de Trichoptera en el Nor- Occidente de Colombia. Caldasia, 25(1), 169-192.

Prather, A. (2004). Revision of the Neotropical caddisfly genus Banyallarga (Trichoptera: Calamoceratidae). Zootaxa, 435, 1-76.

Rincón, M. E. (1996). Aspectos bioecológicos de los tricópteros de la quebrada Carrizal (Boyacá, Colombia). Revista Colombiana de Entomología,22(1), 53-60.

Rivera, O. D. (2001). Páramos de Colombia. Banco de Occidente. Cali, Colombia: I/M Editores.

Roldán, G. (2003). Bioindicación de la calidad del agua en Colombia. Uso del método BMWP/Col. Colombia: Editorial Universidad de Antioquia.

Rös, M., Escobar F. \& Halffter G. (2012). How dung beetles respond to a human- modified variegated landscape in Mexican cloud forest: a study of biodiversity integrating ecological and biogeographical perspectives. Diversity and Distributions, 18, 377-389

Rosenberg, D. M., \& Resh, V. H. (1993). Freshwater Biomonitoring and Benthic macroinvertebrates. New York: Chapman \& Hall.

Springer, M. (2010). Trichoptera. Revista de Biología Tropical, 58(Suppl. 4), 151-198.

Tuomisto, H. (2010). A diversity of beta diversities: straightening up a concept gone awry. Part 1. Defining beta diversity as a function of alpha and gamma diversity. Ecography, 33, 2-22.

Wiggins, G. B. (1996). Trichoptera Families. In R.W. Merritt \& K.W. Cummins (Eds.), An introduction to the aquatic insects of North America, $3^{\text {rd }}$ Ed. (pp. 309-349). Kendal/Hunt Publishing Company, Dubuque, Iowa. 